\title{
Population-based rates, risk factors and consequences of preterm births in South-Asia and sub-Saharan Africa: A multi-country prospective cohort study
}

The Alliance for Maternal and Newborn Health Improvement (AMANHI) Gestational Age Study Group

*Collaborators of the AMANHI Gestational Age Study Group are listed in alphabetical order at the end of the article.

\section{Correspondence to:}

Muhammad Imran Nisar

The Aga Khan University

Stadium Road

PO Box 350-0

Karachi 7480-0

Pakistan

imran.nisar@aku.edu

\section{Sachiyo Yoshida}

Department of Maternal, Newborn, Child and Adolescent Health and

Ageing

World Health Organization

Avenue Appia 20

1202 Genève

Switzerland

yoshidas@who.int
Background Preterm birth is the leading cause of neonatal deaths in low middle-income countries (LMICs), yet there exists a paucity of high-quality data from these countries. Most modelling estimates are based on studies using inaccurate methods of gestational age assessment. We aimed to fill this gap by measuring the population-based burden of preterm birth using early ultrasound dating in five countries in South-Asian and sub-Saharan Africa.

Methods We identified women early in pregnancy ( $<20$ weeks based on last menstrual period) by home visits every 2-3 months (except in Zambia where they were identified at antenatal care clinics) in 5 research sites in South-Asia and sub-Saharan Africa between July 2012 and September 2016. Trained sonographers performed an ultrasound scan for gestational age dating. Women were enrolled if they were 8-19 weeks pregnant on ultrasound. Women $<8$ weeks were rescheduled for repeat scans after 4 weeks, and identified women were followed through pregnancy until 6 weeks postpartum. Site-specific rates and proportions were calculated and a logistic regression model was used to predict the risk factors of preterm birth.

Results Preterm birth rates ranged from 3.2\% in Ghana to $15.7 \%$ in Pakistan. About $46 \%$ of all neonatal deaths occurred among preterm infants, $49 \%$ in South Asia and 40\% in sub-Saharan Africa. Fourteen percent of all preterm infants died during the neonatal period. The mortality was $37.6 \%$ for early preterm babies ( $<34$ weeks), $5.9 \%$ for late preterm babies ( 34 to $<37$ weeks), and $1.7 \%$ for term babies ( 37 to $<42$ weeks). Factors associated lower gestation at birth included South-Asian region (adjusted mean difference (Adj MD) =-6.2 days, 95\% confidence interval $(C I)=-5.5,-6.9)$, maternal morbidities (Adj $\mathrm{MD}=-3.4$ days, 95\% CI=-4.6, -2.2), multiple pregnancies (Adj $\mathrm{MD}=-17.8$ days, 95\% CI=-19.9, -15.8), adolescent pregnancy (Adj $\mathrm{MD}=-2.7$ days, 95\% CI =-3.7, -1.6) and lowest wealth quintile (Adj $\mathrm{MD}=1.3$ days, $95 \% \mathrm{CI}=-2.4,-0.3$ ).

Conclusions Preterm birth rates are higher in South Asia than in sub-Saharan Africa and contribute to $49 \%$ and $40 \%$ of all neonatal deaths in the two regions, respectively. Adolescent pregnancy and maternal morbidities are modifiable risk factors associated with preterm birth.

Preterm birth is a leading cause of death in children under five years of age worldwide. Approximately 14.8 million babies were born preterm in 2014 , accounting for $10.6 \%$ of all live births globally [1]. In addition, about one million babies die due to direct complications of being born preterm, ie, before completing 37 weeks of gestation [2,3]. Globally, the preterm birth rate increased from 9.8\% (8.3-10.9) in 2000 to $10.6 \%$ (9.0-12.0) in 2014. South Asia and sub-Saharan Africa alone contribute to $81.1 \%$ of these preterm births, 
with countries like India, China, Nigeria, Bangladesh, Indonesia and Pakistan having 7.0 million (47.7\%) of preterm births globally in 2014 [1,2].

The cut off for preterm birth defined above is, to an extent, arbitrary. Recent studies show that neonatal outcomes vary within the term group, with greater risks of adverse outcomes associated with early-term neonates than those born at 40 weeks of gestational age [4]. In most low-income settings, where facilities for obstetric ultrasound are not available, the gestational age of the fetus or the newborn is calculated by counting weeks passed since the first day of the last menstrual period (LMP). This is inherently dependent on pregnant women's recall and can be highly inaccurate at times [5,6]. The "gold standard" for measuring gestational age is first-trimester obstetric ultrasound [7-9]. Other methods used to estimate gestational age include symphysis-fundal height, birth weight, and clinical assessment of newborn after birth [10-12] having varying levels of inaccuracy, eg, using birthweight vastly overestimate the number of preterm births, particularly in South-Asian countries where many babies are born small for gestational age (SGA) $[13,14]$.

The preterm birth syndrome involves a complex interplay between various sociodemographic, environmental, and biological factors. Some of the factors identified in the literature include black sub-Saharan African ancestry, family history, prior history of preterm births and an interpregnancy interval of fewer than six months $[2,15]$. Other maternal factors involved are young age, under and over-nutrition, infections, bleeding during pregnancy, and history of substance abuse. In addition, multiple pregnancies carry 10 times the risk of preterm births compared to singleton pregnancies $[15,16]$.

While several studies have showcased global estimates and levels of preterm births, there is a general agreement about the gaps in existing data, particularly from South-Asian and sub-Saharan Africa. One important reason for this disproportionate reporting of data are absent or incomplete registries [17]. Even though WHO guidelines recommend antenatal ultrasounds for all pregnant women, this is rarely practiced for various reasons [18]. There is also misclassification of live births, stillbirths, and deaths which affect reporting of preterm births, including various phenotypes such as spontaneous or provider-initiated in these regions [1]. In the given scenario, it is imperative to generate accurate data on the true burden of preterm births and its associated risk factors. This will help us better understand preterm birth and develop effective primary and secondary interventions to decrease the associated morbidity and mortality.

\section{METHODS}

The Alliance for Maternal and Newborn Health Improvement (AMANHI) gestational age group conducted a multi-country, population-based prospective cohort study to evaluate the diagnostic accuracy of simple methods for gestational age assessment (including reported LMP, physical, neuromuscular, feeding assessments, and anthropometry). These methods were compared with gestational age calculated from early pregnancy ultrasound scans. The study protocol was previously published [19].

We conducted a study in five community sites in South-Asia (Sylhet, Bangladesh, and Karachi, Pakistan), sub-Saharan Africa (Kintampo, Ghana; Pemba, Tanzania; and Southern Province, Zambia) between July 2012 and September 2016. The detailed methods for enrollment and follow up are described elsewhere [20]. Briefly, in 4 out of 5 sites, pregnant women were identified at home using 2 to 3 monthly household surveillance, offered a pregnancy test, and a gold standard ultrasound scan for gestational age dating. Sonographers at all sites received centralized training coordinated by the WHO MCA department. Women were enrolled if they were between 8-19 weeks pregnant on ultrasound. Women $<8$ weeks were rescheduled for repeat scans after 4 weeks and enrolled at $\geq 8$ weeks gestation. At 20 weeks or more, pregnant women were counselled to continue routine antenatal clinic attendance but were not enrolled on the AMANHI gestational age study.

We measured only the crown-rump length (CRL) if the pregnancy was less than 14 weeks (CRL<95mm), both biparietal diameter (BPD), and femur length (FL) if more than 14 weeks, and all three if within the 14th week. Thus, we conducted three measurements for each biometric parameter. We promptly referred women with major abnormalities or intrauterine fetal deaths to health facilities for appropriate management. The preterm birth rate was defined as all live births before 37 completed weeks of gestation divided by the total number of live births. Early to moderate preterm birth rate was defined as live births before 34 completed weeks of gestation, divided by the total live births. Small for gestational age was defined as newborns who were less than 10th centile for their gestational age using INTERGROWTH criterion [20].

We established a birth notification system to capture birth outcomes as close to birth as possible, which included active surveillance, reporting by family members and key informants. All live births were then followed till 
28 days of life to ascertain vital status. In case mortality was recorded, we conducted a detailed verbal autopsy interview after a culturally appropriate mourning period.

Data were collected on paper and entered electronically at all sites except Zambia, where filled forms were scanned using TeleForms Software (Hewlett Packard, Sunnyvale, CA). Sites shared data to WHO quarterly for quality assurance and data monitoring. Any inconsistencies were flagged and resolved in consultation with data managers and investigators from the sites.

Logistic regressions were performed to examine the association of gestational age and neonatal mortality and identify risk factors of preterm birth. A multivariate model was adjusted for variables with a $P$ value of $\leq 0 \cdot 10$ at the univariate model and the site. In the analysis on the risk factor of preterm birth, we used different hierarchical models to obtain adjusted odds rations (ORs) [21]. We calculated the mean difference in gestation for different factors associated with the duration of gestation among women with the first postnatal visit. This analysis performed the imputation of missing values by mean imputation for height and the frequent category for all other covariates. The site was put in as a factor variable. Site interactions were tested to identify variables whose effects might vary across sites. Site-specific gestational age distributions were plotted using both LMP and gold standard ultrasound. A Bland Altman plot was created for the difference in gestational age estimates between the gold standard and the LMP (Ultrasound based gestational age-LMP). All analysis was carried out using Stata version 16 (Stata Corp, College Station, TX, USA).

\section{Ethical approval}

Ethical approval was obtained from WHO Ethical Review Committee (ERC) and all site ERC's. Fieldworkers obtained consent from pregnant women in their local or preferred language.

\section{RESULTS}

Of 13814 women who had an ultrasound for gestational age assessment, 11662 (84\%) were enrolled. Of them, 10763 (92\%) women were followed up till birth. There were 10581 live births registered in the study, of which 9974 were included in the analysis (Figure 1). Vital status at the end of the neonatal period was known for

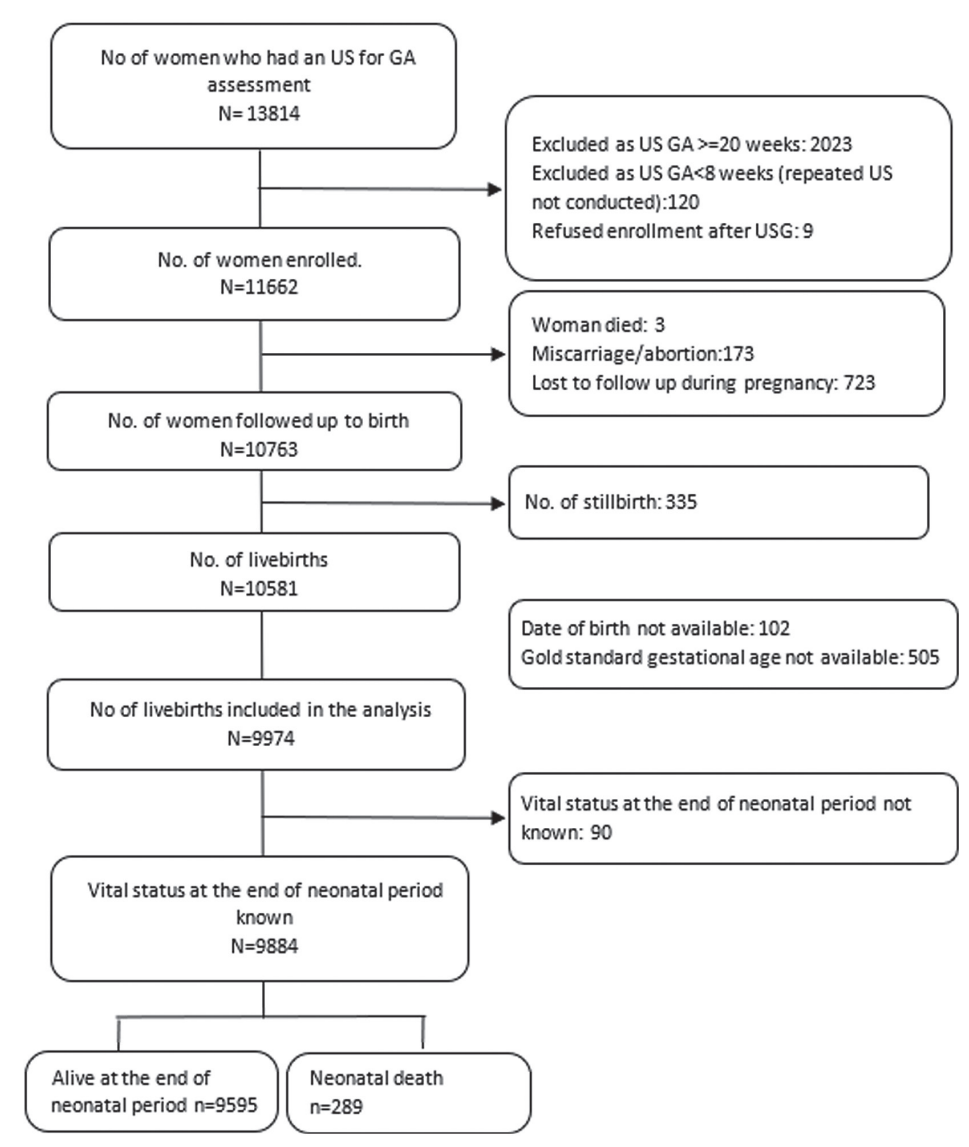

Figure 1. Flowchart of study participants (all sites combined). 9884 live births. Baseline characteristics of enrolled women with the known birth outcome are described for each site (Table 1). Most pregnant women were in the age group 20-34 years old. The level of their education varied between the sites, with more than half of women in Pakistan (59.2\%) having no formal education, to the majority having some level of education (86\%) in Zambia. The highest previous child death rate was reported in Ghana (21.7\%), the highest previous stillbirth rate in Bangladesh (13.1\%) and the highest previous preterm rate was reported by mothers in Zambia (3.7\%). South-Asian women were on average shorter in height as compared to their sub-Saharan African counterparts. Antepartum haemorrhage ranged from a low of $0.3 \%$ of women in Bangladesh to a high of $3.6 \%$ in Pakistan. Similarly, later antepartum infection (fever before or during delivery) was highest in the Pakistan site (12.4\%). Pre-eclampsia or eclampsia was present in less than $1 \%$ of women in all sites except in Tanzania (4.7\%). The rate of multiple gestations was highest in Ghana and Tanzania (3.9\%). Majority of women in sub-Saharan African sites delivered at a health facility in the presence of a skilled birth attendant, while only $49.0 \%$ of births in Bangladesh and $65.3 \%$ in Pakistan occurred at a health care facility. The proportion of women having normal vaginal deliveries ranged from (85.4\%-96.7\%). Of the 9173 term births ( $\geq 37$ weeks), $2.0 \%$ resulted in stillbirth, and of 1114 preterm birth ( $<37$ weeks), $11.9 \%$ resulted in stillbirth (data not shown). 
Table 1. Baseline characteristics of enrolled pregnant women $(n=10763)$

\begin{tabular}{|c|c|c|c|c|c|}
\hline & \multicolumn{2}{|c|}{ SOUTH ASIAN SITES } & \multicolumn{3}{|c|}{ SUB-SAHARAN AFRICAN SITES } \\
\hline & $\begin{array}{c}\text { Bangladesh } \\
(\mathrm{N}=2982)\end{array}$ & $\begin{array}{c}\text { Pakistan } \\
(\mathrm{N}=2608)\end{array}$ & $\begin{array}{l}\text { Ghana } \\
(\mathrm{N}=976)\end{array}$ & $\begin{array}{c}\text { Tanzania } \\
(\mathrm{N}=2427)\end{array}$ & $\begin{array}{c}\text { Zambia } \\
(\mathrm{N}=981)\end{array}$ \\
\hline \multicolumn{6}{|l|}{ Characteristics of woman and family: } \\
\hline Pregnant woman's age, $\mathrm{n}(\%)$ & 2331 & 2171 & 970 & 2420 & 962 \\
\hline $15-19$ & $458(19.7)$ & $163(7.5)$ & $85(8.8)$ & $187(7.7)$ & $276(28.7)$ \\
\hline $20-34$ & $1799(77.2)$ & $1788(82.4)$ & $735(75.8)$ & $1764(72.9)$ & $579(60.2)$ \\
\hline $35+$ & $74(3.2)$ & $220(10.1)$ & $150(15.5)$ & $469(19.4)$ & $107(11.1)$ \\
\hline Woman's education, n (\%) & 2891 & 2171 & 970 & 2420 & 956 \\
\hline None & $249(8.6)$ & $1285(59.2)$ & $280(28.9)$ & $310(12.8)$ & $18(1.9)$ \\
\hline $1-6 y$ & $1207(41.8)$ & $358(16.5)$ & $597(61.6)$ & $832(34.4)$ & $97(10.2)$ \\
\hline $7-12 y$ & $1415(49)$ & $510(23.5)$ & $77(7.9)$ & $1246(51.5)$ & $822(86)$ \\
\hline $13+$ years & $20(0.7)$ & $18(0.8)$ & $16(1.7)$ & $32(1.3)$ & $19(2)$ \\
\hline Previous child death n (\%) & 2005 & 1731 & 801 & 1895 & 674 \\
\hline$\geq 1$ child death & $393(19.6)$ & $154(8.9)$ & $174(21.7)$ & $299(15.8)$ & $90(13.4)$ \\
\hline Previous stillbirth, n (\%) & 2005 & 1905 & 801 & 1967 & 674 \\
\hline$\geq 1$ stillbirth & $262(13.1)$ & $160(8.4)$ & $74(9.2)$ & $176(9)$ & $16(2.4)$ \\
\hline Previous preterm birth, n (\%) & 2001 & 1892 & 801 & 1895 & 672 \\
\hline Yes & $30(1.5)$ & $48(2.5)$ & $13(1.6)$ & $34(1.8)$ & $25(3.7)$ \\
\hline Maternal height, n (\%) & 2156 & 953 & 798 & 2121 & 868 \\
\hline Mean (SD) & $150.1(5.1)$ & $155.1(5.9)$ & $158.0(6.9)$ & $155.2(6.4)$ & $160.2(7.6)$ \\
\hline \multicolumn{6}{|l|}{ Household characteristics: } \\
\hline Clean cooking fuel, n (\%) & 2914 & 2171 & 969 & 2420 & 954 \\
\hline Yes & $34(1.2)$ & $1953(90)$ & $100(10.3)$ & $350(14.5)$ & $66(6.9)$ \\
\hline Improved latrine facility*, n (\%) & 2977 & 2171 & 969 & 2420 & 961 \\
\hline Yes & $2876(96.6)$ & $2085(96)$ & $768(79.3)$ & $1781(73.6)$ & $889(92.5)$ \\
\hline Wealth quintile, n (\%) & 2971 & 2171 & 970 & 2420 & 622 \\
\hline Poorest & $560(18.9)$ & $429(19.8)$ & $196(20.2)$ & $488(20.2)$ & $131(21.1)$ \\
\hline Poorer & $597(21.0)$ & $435(20.0)$ & $207(21.3)$ & $512(21.2)$ & $103(16.6)$ \\
\hline Middle & $604(20.3)$ & $434(20.0)$ & $188(19.4)$ & $485(20.0)$ & $120(19.3)$ \\
\hline Richer & $616(20.7)$ & $428(19.7)$ & $188(19.4)$ & $461(19.1)$ & $124(19.9)$ \\
\hline Richest & $594(20.0)$ & $445(20.5)$ & $191(19.7)$ & $474(19.6)$ & $144(23.2)$ \\
\hline Piped drinking water access, n (\%) & 2976 & 2171 & 970 & 2420 & 956 \\
\hline Yes & $35(1.2)$ & $971(44.7)$ & $514(53.0)$ & $2245(92.8)$ & $255(26.7)$ \\
\hline \multicolumn{6}{|c|}{ Morbidity during the current pregnancy: } \\
\hline Antepartum hemorrhage, n(\%) & 2303 & 1718 & 974 & 2406 & 968 \\
\hline Yes & $9(0.3)$ & $61(3.6)$ & $26(2.7)$ & $26(1.1)$ & $7(0.7)$ \\
\hline Pre-eclampsia or eclampsia, n(\%) & 2301 & 1712 & 974 & 2406 & 968 \\
\hline Yes & $15(0.7)$ & $11(0.8)$ & $8(0.8)$ & $112(4.7)$ & $0(0)$ \\
\hline Fever before or during delivery, n (\%) & 2139 & 1363 & 969 & 2326 & 566 \\
\hline Yes & $33(1.5)$ & $169(12.4)$ & $57(5.9)$ & $12(0.5)$ & $0(0)$ \\
\hline \multicolumn{6}{|l|}{ Birth characteristics: } \\
\hline Multiple birth, n (\%) & 2983 & 2608 & 976 & 2427 & 981 \\
\hline Yes & $24(0.8)$ & $69(2.7)$ & $38(3.9)$ & $95(3.9)$ & $20(2)$ \\
\hline Childbirth in a health facility, n (\%) & 2845 & 2608 & 973 & 2329 & 828 \\
\hline Yes & $1395(49)$ & $1699(65.3)$ & $784(80.6)$ & $2324(99.8)$ & $710(85.8)$ \\
\hline Skilled birth attendant, n (\%) & 2844 & 2604 & 973 & 2329 & 807 \\
\hline Yes & $1355(47.6)$ & $1816(69.7)$ & $770(79.1)$ & $1716(73.7)$ & $649(80.4)$ \\
\hline Type of delivery, n (\%) & 2845 & 2,508 & 973 & 2329 & 858 \\
\hline Normal vaginal delivery & $2449(86.1)$ & $2141(85.4)$ & $848(87.2)$ & $2241(96.2)$ & $830(96.7)$ \\
\hline Assisted vaginal delivery & $48(1.7)$ & $47(1.9)$ & $8(0.8)$ & $8(0.3)$ & $3(0.4)$ \\
\hline C-section & $348(12.2)$ & $320(12.8)$ & 117 (12) & 80 (3.4) & $25(2.9)$ \\
\hline
\end{tabular}

$\mathrm{CI}$ - confidence interval, SD - standard deviation

*Flush/pour flush toilet. 
Among the 9974 live births across five sites, preterm birth rates ranged from 3.2\% in Ghana to $15.7 \%$ in Pakistan (Table 2). More than one in four infants were born with low birth weight $(<2500 \mathrm{~g})$ in South-Asian sites (26.6\% in Bangladesh, 25.2\% in Pakistan). Similarly, infants who were small for gestational age were more in South-Asian sites (42.7\% in Bangladesh and 35.5\% in Pakistan) than in sub-Saharan African sites (10.2\% in Tanzania and 19.2\% in Zambia) except in Ghana, showing a similar rate as for Pakistan (34.3\%).

Table 2. Gestation and size at the time of birth for live-born infants $(n=9974)$

\begin{tabular}{|c|c|c|c|c|c|}
\hline & \multicolumn{2}{|c|}{ SOUTH ASIAN SITES } & \multicolumn{3}{|c|}{ SUB-SAHARAN AFRICAN SITES } \\
\hline & $\begin{array}{l}\text { Bangladesh } \\
(\mathrm{N}=2982)\end{array}$ & $\begin{array}{l}\text { Pakistan } \\
(\mathrm{N}=2608)\end{array}$ & $\begin{array}{l}\text { Ghana } \\
(\mathrm{N}=976)\end{array}$ & $\begin{array}{l}\text { Tanzania } \\
(\mathrm{N}=2427)\end{array}$ & $\begin{array}{c}\text { Zambia } \\
(\mathrm{N}=981)\end{array}$ \\
\hline \multicolumn{6}{|l|}{ Gestational age at birth* } \\
\hline Mean (SD) & $38.9(2.1)$ & $38.6(2.1)$ & $39.9(1.6)$ & $39.5(1.7)$ & $39.8(2.8)$ \\
\hline Median (IQR) & $\begin{array}{c}39.3 \\
(38.1,40.1) \\
\end{array}$ & $\begin{array}{c}38.9 \\
(37.7,39.9) \\
\end{array}$ & $\begin{array}{c}40 \\
(39.1,40.9) \\
\end{array}$ & $\begin{array}{c}39.6 \\
(38.7,40.4) \\
\end{array}$ & $\begin{array}{c}39.9 \\
(38.7,40.9) \\
\end{array}$ \\
\hline Preterm birth* (<37 wks) & $\begin{array}{c}11.7 \% \\
(10.6 \%, 12.9 \%) \\
\end{array}$ & $\begin{array}{c}15.7 \% \\
(14.3 \%, 17.1 \%)\end{array}$ & $\begin{array}{c}3.2 \% \\
(2.3 \%, 4.6 \%) \\
\end{array}$ & $\begin{array}{c}4.9 \% \\
(4.1 \%, 5.8 \%) \\
\end{array}$ & $\begin{array}{c}7.4 \% \\
(5.9 \%, 9.3 \%) \\
\end{array}$ \\
\hline Gestational age at birth categories (\%)* & 2982 & 2608 & 976 & 2427 & 981 \\
\hline Early preterm $(<34$ wk $)$ & $99(3.3 \%)$ & $79(3 \%)$ & $5(0.5 \%)$ & $31(1.3 \%)$ & $30(3.1 \%)$ \\
\hline Late Preterm ( 34 to $<37 \mathrm{wk}$ ) & $250(8.4 \%)$ & $330(12.7 \%)$ & $27(2.8 \%)$ & $88(3.6 \%)$ & $43(4.4 \%)$ \\
\hline Term (37 to $<42 \mathrm{wk})$ & $2560(85.2 \%)$ & $2172(83.3 \%)$ & $887(90.9 \%)$ & $2220(91.5 \%)$ & $793(80.8 \%)$ \\
\hline Post term ( $\geq 42 \mathrm{wk})$ & $74(2.5 \%)$ & $27(1.0 \%)$ & $57(5.8 \%)$ & $88(3.6 \%)$ & $115(11.7 \%)$ \\
\hline Mean birth weight in grams $\dagger$ & $2752(488)$ & $2764(492)$ & $2940(456)$ & $3283(521)$ & $3107(511)$ \\
\hline Low birth weight $(<2500 \mathrm{~g})(\%, 95 \% \mathrm{CI})$ & $\begin{array}{c}26.6 \% \\
(24.9 \%, 28.3 \%) \\
\end{array}$ & $\begin{array}{c}25.2 \% \\
(23.5 \%, 27.0 \%) \\
\end{array}$ & $\begin{array}{c}13.2 \% \\
(11.1 \%, 15.5 \%)\end{array}$ & $\begin{array}{c}6.4 \% \\
(5.4 \%, 7.5 \%) \\
\end{array}$ & $\begin{array}{c}7.9 \% \\
(6.2 \%, 10.0 \%) \\
\end{array}$ \\
\hline Birth weight categories in grams, $n(\%)$ & 2590 & 2415 & 970 & 2320 & 787 \\
\hline$<1500 \mathrm{~g}$ & $14(0.5 \%)$ & $18(0.8 \%)$ & $4(0.4 \%)$ & $4(0.2 \%)$ & $2(0.3 \%)$ \\
\hline 1500-1999g & $82(3.2 \%)$ & $108(4.5 \%)$ & $12(1.2 \%)$ & $29(1.3 \%)$ & $8(1.0 \%)$ \\
\hline $2000-2499 \mathrm{~g}$ & $592(22.9 \%)$ & $482(20.0 \%)$ & $112(11.6 \%)$ & $115(5.0 \%)$ & $52(6.6 \%)$ \\
\hline 2500-2999g & $1142(44.1 \%)$ & 1099 (45.5\%) & $361(37.2 \%)$ & $472(20.3 \%)$ & $220(28.0 \%)$ \\
\hline$\geq 3000 \mathrm{~g}$ & $760(29.3 \%)$ & $708(29.3 \%)$ & $481(49.6 \%)$ & $1700(73.3 \%)$ & $505(64.2 \%)$ \\
\hline Small for gestational age $\neq, \%(95 \%$ CI) & $\begin{array}{c}42.7 \% \\
(40.8 \%, 44.7 \%) \\
\end{array}$ & $\begin{array}{c}35.5 \% \\
(33.6 \%, 37.4 \%)\end{array}$ & $\begin{array}{c}34.3 \% \\
(31.4 \%, 37.4 \%)\end{array}$ & $\begin{array}{c}10.2 \% \\
(9.0 \%, 11.5 \%)\end{array}$ & $\begin{array}{c}19.2 \% \\
(16.5 \%, 22.2 \%)\end{array}$ \\
\hline
\end{tabular}

\section{CI - confidence interval}

*Gestational age is based on ultrasound (gold standard).

$\dagger$ Birth weight is missing for 392 (13.2\%) in Bangladesh, 193 (7.4\%) in Pakistan, 6 (0.6\%) in Ghana, 107 (4.4\%) in Tanzania and 194 (19.8\%) in Zambia.

$¥$ Size for gestational age is missing for 441 (14.8\%) in Bangladesh, 203 (7.8\%) in Pakistan, 23 (2.4\%) in Ghana, 125 (5.2\%) in Tanzania and $236(24.1 \%)$ in Zambia.

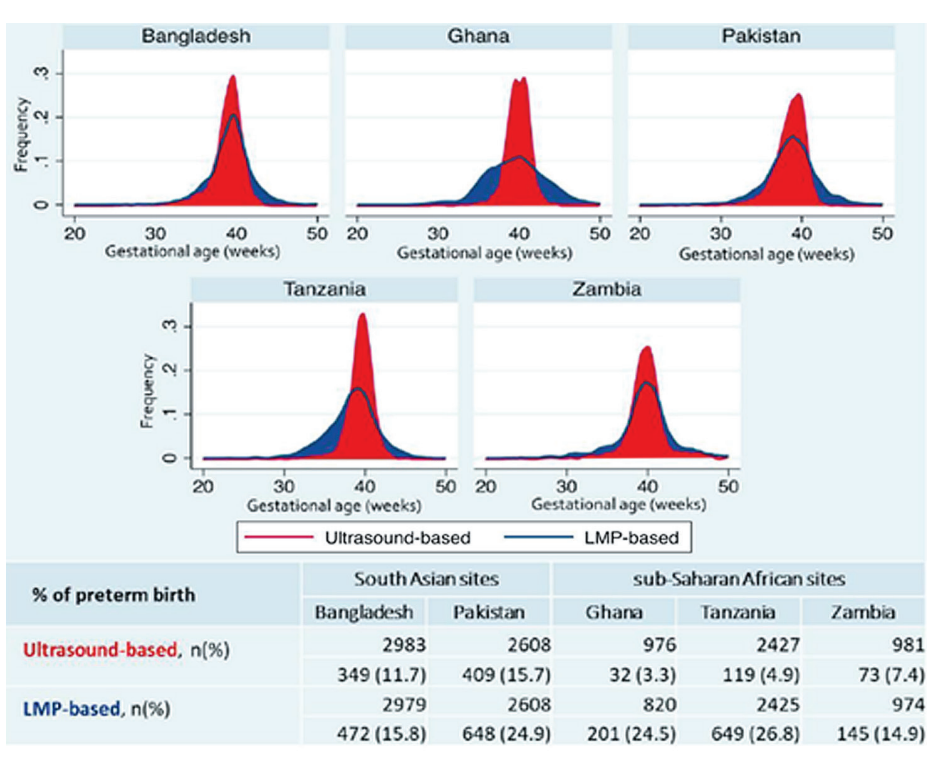

Figure 2. Frequency of gestational age in weeks by gestational age assessment method and site.
Estimates for preterm birth rate were much higher than ultrasound-based gestational age when LMP was used for estimation (Figure 2). The difference in the burden of preterm birth by two measures was more pronounced in sub-Saharan African sites. However, the LMP based preterm birth was similar in the two regions.

Comparison of gestational age at the time of ultrasound by two measurements (ultrasound vs LMP) showed that the 95\% limits of agreement ranged from -35.2 to 39.1 days (Mean bias $=1.95$ days; standard deviation $(S D)=18.95)$ (Figure S1 in the Online Supplementary Document). Thus, LMP tends to overestimate gestational age at earlier gestation and underestimate at a later gestation.

Of the 9884 live births, 289 (2.9\%) resulted in neonatal deaths (Table 3$)$. The neonatal mortality rate was slightly higher in South-Asian sites than in sub-Saharan African sites (3.6\% in South-Asian sites and 2.0\% 
Table 3. Neonatal deaths by gestational age at birth, overall and by region

\section{OVERALL}

Live births whose vital status at the end of neonatal period is known

Neonatal deaths

9884

Proportion of preterm deaths among all neonatal deaths

$289(29 / 1000$ LB $)$

Proportion of preterm infants who died during neonatal period

Gestational age* in completed week (gestational age available $\mathrm{n}=9884$ )

\begin{tabular}{|c|c|c|c|c|}
\hline$<32$ weeks $(\mathrm{n}=98)$ & $61(62.2 \%)$ & $98.4(63.3,152.9)$ & $108.2(52.1,224.1)$ & \\
\hline 32 to $<34$ weeks $(n=144)$ & $30(20.8 \%)$ & $15.7(10.2,24.3)$ & $13.7(6.9,27.2)$ & \\
\hline 34 to $<37$ weeks $(\mathrm{n}=732)$ & $43(5.9 \%)$ & $3.7(2.6,5.3)$ & $3.6(2.1,6.3)$ & $\mathrm{P}<0.001$ \\
\hline 37 to $<42$ weeks $(\mathrm{n}=8556)$ & $141(1.7 \%)$ & 1.0 (reference) & 1.0 (reference) & \\
\hline$\geq 42$ weeks $(n=354)$ & $14(4.0 \%)$ & $2.5(1.4,4.3)$ & $1.9(0.67,5.4)$ & \\
\hline \multicolumn{5}{|l|}{ ASIA } \\
\hline Live births whose vital status at the end of neonatal period is known & & 5563 & & \\
\hline Neonatal deaths & \multicolumn{4}{|c|}{$201(36.1 / 1000 \mathrm{LB})$} \\
\hline Proportion of preterm deaths among all neonatal deaths & \multicolumn{4}{|c|}{$49.3 \%$} \\
\hline Proportion of preterm infants who died during neonatal period & \multicolumn{4}{|c|}{$13.2 \%$} \\
\hline gestational age* in week (gestational age available $\mathrm{n}=5563$ ) & Neonatal deaths & OR $(95 \% \mathrm{CI})$ & $\begin{array}{l}\text { Adjusted OR } \dagger \\
(95 \% \mathrm{CI})\end{array}$ & P-value \\
\hline$<32$ weeks $(n=69)$ & $45(65.2 \%)$ & $93.1(54.4,159,2)$ & $162.2(61.5,427.7)$ & \\
\hline 32 to $<34$ weeks $(n=109)$ & $18(16.5 \%)$ & $9.8(5.7,17.0)$ & $9.8(4.1,23.5)$ & \\
\hline 34 to $<37$ weeks $(n=574)$ & $36(6.3 \%)$ & $3.3(2.2,4.9)$ & $4.0(2.1,7.6)$ & $\mathrm{P}<0.001$ \\
\hline 37 to $<42$ weeks $(n=4712)$ & $93(2.0 \%)$ & 1.0 (reference) & 1.0 (reference) & \\
\hline$\geq 42$ weeks $(n=99)$ & $9(9.1 \%)$ & $4.7(2.4,10.2)$ & $3.0(0.7,13.2)$ & \\
\hline \multicolumn{5}{|l|}{ AFricA } \\
\hline Live births whose vital status at the end of the neonatal period is known & & 4321 & & \\
\hline Neonatal deaths & \multicolumn{4}{|c|}{$88(20.4 / 1000$ LB) } \\
\hline Proportion of preterm deaths among all neonatal deaths & \multicolumn{4}{|c|}{$39.8 \%$} \\
\hline Proportion of preterm infants who died during neonatal period & \multicolumn{4}{|c|}{$15.8 \%$} \\
\hline Gestational age* in week (gestational age available $\mathrm{n}=4321$ ) & Neonatal deaths & OR (95\% CI) & $\begin{array}{l}\text { Adjusted OR } \dagger \\
(95 \% \mathrm{CI})\end{array}$ & $\boldsymbol{P}$-value \\
\hline$<32$ weeks $(n=29)$ & $16(55.2 \%)$ & $97.3(44.4,213.5)$ & $65.7(17.8,242.2)$ & \\
\hline 32 to $<34$ weeks $(n=35)$ & $12(34.3 \%)$ & $41.3(19.4,87.7)$ & $39.5(10.6,146.0)$ & \\
\hline 34 to $<37$ weeks $(n=158)$ & $7(4.4 \%)$ & $3.7(1.6,8.2)$ & $2.7(0.8,9.3)$ & $\mathrm{P}<0.001$ \\
\hline 37 to $<42$ weeks $(n=3844)$ & $48(1.3 \%)$ & 1.0 (reference) & 1.0 (reference) & \\
\hline$\geq 42$ weeks $(n=255)$ & $5(2.0 \%)$ & $1.6(0.6,4.0)$ & $1.3(0.3,5.4)$ & \\
\hline
\end{tabular}

OR - odds ratio, $\mathrm{CI}$ - confidence interval

*Gestational age is based on ultrasound.

†OR was adjusted for site, mother's age, mother's education, wealth quintile and previous obstetric history, including previous stillbirth and previous preterm birth, morbidity during pregnancy (antepartum haemorrhage, pre-eclampsia and eclampsia, antepartum fever) and multiple gestations.

in sub-Saharan African sites). Infants born preterm accounts for approximately $46 \%$ of neonatal deaths (49\% in South-Asia and $40 \%$ in sub-Saharan African sites). About 16\% of preterm infants in sub-Saharan African sites and 13\% in South-Asian sites died during the neonatal period.

The risk of neonatal deaths in very preterm infants ( $<32$ weeks) was $62.2 \%$ (adjusted odds ratio $(\mathrm{aOR})=108.2$, 95\% confidence interval $(\mathrm{CI})=52.1,224.1$; South-Asia $\mathrm{aOR}=162.0,95 \% \mathrm{CI}=61.5,427.7$; sub-Saharan Africa $\mathrm{aOR}=65.7,95 \% \mathrm{CI}=17.8,242.2$ ). Risk of neonatal mortality in moderate preterm infants (32 to $<34$ weeks) was 20.8\% (aOR=13.7, 95\% CI=6.9, 27.2; South-Asia aOR=9,8, 95\% CI=4.1, 23.5; $P<0.001$; sub-Saharan Africa $\mathrm{aOR}=39.5,95 \% \mathrm{CI}=10.6,146.0 ; \mathrm{P}<0.001)$ (Table 3; Figure 3).

The mean duration of gestation was 274.6 days ( $S D=13.8$ ), and the duration was shorter by 6.2 days in SouthAsian sites than in sub-Saharan African sites (adjusted mean difference $(\mathrm{aMD})=6.2$ days, $95 \% \mathrm{CI}=5.5,6.9$ ) (Table 4). Mean gestational age at birth was 18.1 days shorter in case of multiple pregnancies ( $\mathrm{aMD}=-17.8$, 95\% CI $=-19.9,-15.8$ ), adolescent pregnancy ( $\mathrm{aMD}=-2.7$ days, $95 \% \mathrm{CI}=-3.7,-1.6$ ) and low socioeconomic status (poorest wealth quintile) ( $\mathrm{aMD}=-1.3$ days, 95\% $\mathrm{CI}=-2.4,-0.3$ ).

Multivariate analyses examining the risk factor of preterm birth (all preterm and spontaneous preterm) showed consistent results (Tables S1 and S2 in the Online Supplementary Document). The factors associated with preterm birth included: younger maternal age group of $15-19$ years $(\mathrm{aOR}=1.27,95 \% \mathrm{CI}=1.03,1.58)$, low 


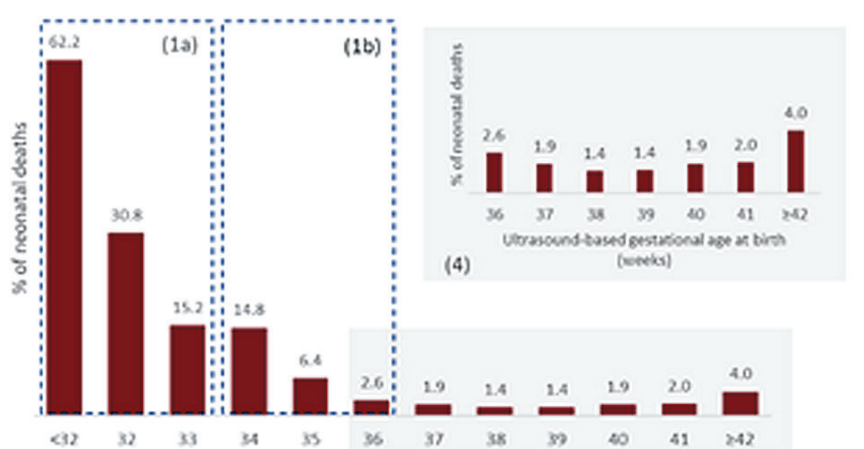

Ultrasound-based gestational age at birth (in weeks)

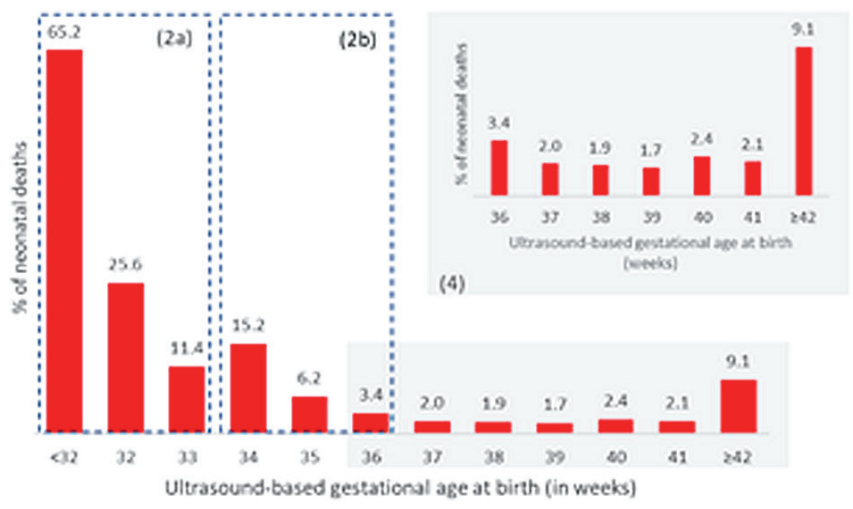

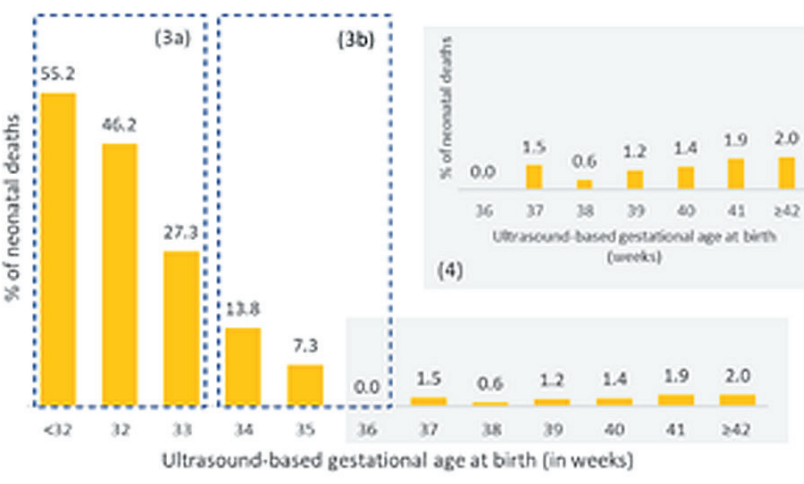

Overall Asia

(1a) Overall: $2.5 \%$ of births; $32 \%$ of neonatal deaths (1b) Overall: $7.4 \%$ of births; $15 \%$ of neonatal deaths (2a) Asia: $3.2 \%$ of births; $31 \%$ of neonatal deaths (2b) Asia: $10.4 \%$ of births; $18 \%$ of neonatal deaths (3a) Africa: $1.5 \%$ of births: $32 \%$ of neonatal deaths (3b) Africa: $3.6 \%$ of births; $8 \%$ of neonatal deaths

(4) Enlarged graph of neonatal deaths of babies with gestational age at birth of 36 weeks $\propto$ more

Figure 3. Risk of neonatal death by gestational age, overall and by region.

Table 4. Explanatory factor associated with duration of gestation, among women with the first postnatal visit $(n=7031)$

\begin{tabular}{|c|c|c|c|}
\hline \multirow{2}{*}{$\begin{array}{l}\text { ULTRASOUND-BASED GESTATIONAL AGE } \\
\text { Mean (SD)- in days* }\end{array}$} & \multicolumn{3}{|c|}{ OVERALL (N = 7031) } \\
\hline & \multicolumn{3}{|c|}{$274.6(13.8)$} \\
\hline \multirow{2}{*}{ EXPLANATORY FACTORS } & \multicolumn{3}{|c|}{ EFFECT SIZE } \\
\hline & $\mathrm{n} / \mathrm{N}(\%)$ & $\operatorname{MD}(95 \% \mathrm{CI}) \dagger$ & $\operatorname{AdjMD}(95 \% \mathrm{CI}) \ddagger$ \\
\hline \multicolumn{4}{|l|}{ Region: } \\
\hline Asia (ref) & $3162 / 7031(45.0)$ & 1 & 1 \\
\hline Africa & $3869 / 7031(55.0)$ & $6.2(5.5 ; 6.8)$ & $6.2(5.5 ; 6.9)$ \\
\hline Cesarean section (ref: vaginal birth) & $610 / 7017(8.7)$ & $-0.6(-1.8 ; 0.5)$ & $-0.4(-1.5 ; 0.8)$ \\
\hline \multicolumn{4}{|l|}{ Obstetric history: } \\
\hline Primipara & $1537 / 6910(22.2)$ & $-0.4(-1.1 ; 0.4)$ & $0.6(-0.3 ; 1.4)$ \\
\hline Multipara with previous stillbirth & $469 / 6910(6.8)$ & $-1.7(-3.0 ;-0.4)$ & $-1.2(-2.5 ; 0.0)$ \\
\hline $\begin{array}{l}\text { Multipara with previous preterm birth or with previous stillbirth } \\
\text { and previous preterm birth }\end{array}$ & $99 / 6910(1.4)$ & $0.9(-3.6 ; 1.8)$ & $-0.8(-3.4 ; 1.9)$ \\
\hline Multipara with no previous stillbirth or preterm birth (ref) & $4805 / 6910(69.5)$ & 1 & 1 \\
\hline Morbidity during pregnancy (ref: no)§ & $509 / 7009(7.3)$ & $-3.8(-5.0 ;-2.6)$ & $-3.4(-4.6 ;-2.2)$ \\
\hline Multiple pregnancy (ref: singleton pregnancy) & $163 / 6868(2.3)$ & $-18.1(-20.2 ;-16.1)$ & $-17.8(-19.9 ;-15.8)$ \\
\hline Adolescent (15-19y) (ref: >19 y) & $900 / 7015(12.8)$ & $-2.0(-2.9 ;-1.0)$ & $-2.7(-3.7 ;-1.6)$ \\
\hline No education (ref: some education) & $1508 / 6994(21.6)$ & $-0.3(-1.1 ; 0.5)$ & $0.1(-0.7 ; 0.9)$ \\
\hline Maternal height $($ per $\mathrm{cm}) \|$ & $154.5(7.1)$ & $0.1(0.0 ; 0.1)$ & $0.1(0.0 ; 0.1)$ \\
\hline \multicolumn{4}{|l|}{ Wealth quintile: } \\
\hline Poorest & $1412 / 7010(20.1)$ & $-1.4(-2.4 ;-0.4)$ & $-1.3(-2.4 ;-0.3)$ \\
\hline Poor & $1416 / 7010(20.2)$ & $-1.2(-2.2 ;-0.2)$ & $-0.9(-1.9 ; 0.1)$ \\
\hline Middle & $1416 / 7010(20.2)$ & $-0.7(-1.7 ; 0.3)$ & $-0.6(-1.6 ; 0.4)$ \\
\hline Rich & $1363 / 7010(19.4)$ & $-0.4(-1.4 ; 0.6)$ & $-0.3(-1.3 ; 0.7)$ \\
\hline Richest (ref) & $1403 / 7010(20.0)$ & 1 & 1 \\
\hline
\end{tabular}

MD - mean difference

*Gestational age at birth in days by site [mean(sd)]: Asia: Bangladesh 271.9 (14.5), Pakistan 270.2 (14.2); Africa: Ghana 279.5 (10.9), Tanzania 276.3 (11.8), Zambia 278.1 (17.6).

$\uparrow$ Adjusted for region.

¥Adjusted for type of delivery, maternal age, maternal education, wealth quintile, multiple births, obstetric history, and any maternal morbidity (eclampsia or pre-eclampsia, antepartum hemorrhage, fever.

§Morbidity during pregnancy includes pre-eclampsia or eclampsia or fever before delivery or antepartum hemorrhage.

\|l Mean. 
socioeconomic status $(\mathrm{aOR}=1.3695 \%, 95 \% \mathrm{CI}=1.05,1.77)$, history of the previous stillbirth $(\mathrm{aOR} 1.84,95 \%$ $\mathrm{CI}=1.42,2.37$ ) and previous preterm birth (AdjOR 1.96, 95\% $\mathrm{CI}=1.18,3.26$ ). Other pregnancy-related risk factors included pre-eclampsia /eclampsia $(\mathrm{aOR}=2.74,95 \% \mathrm{CI}=1.56,4.84)$, fever before or during delivery $(\mathrm{aOR}=1.76,95 \% \mathrm{CI}=1.18,2.62)$, and multiple gestations $(\mathrm{aOR}=14.3,95 \% \mathrm{CI}=9.92,20.52)$.

\section{DISCUSSION}

This was a large population-based study reporting preterm birth rates across South-Asian and sub-Saharan African sites using gestational age based on ultrasound scan. In our study, we found that preterm birth rates were much higher in South-Asian sites than in sub-Saharan African sites, from 15.7\% in Pakistan to 3.2\% in Ghana.

We found higher proportions of LBW and SGA babies in South-Asian sites. This could be due to the poor nutritional status of these women. Nearly a quarter of women were underweight in South-Asian sites while only 5.8\% in sub-Saharan African sites in AMANHI biorepository cohort- unpublished data. Micronutrient and protein-energy supplementation provided to pregnant mothers seem to significantly reduce SGA births and low birth weights $[22,23]$. Unfortunately, we did not collect information on maternal nutritional status in this study. Maternal height can be another independent risk factor. A previously published meta-analysis showed that a large proportion of preterm birth is attributable to short maternal stature $(31.2 \%$ of preterm births in South-Asia and 10.4\% of preterm births in sub-Saharan Africa) [24]. Other studies have also concluded that shorter mothers deliver babies prematurely with lower birth weights [24,25]. In our study, and on average, women in Bangladesh were $10.0 \mathrm{~cm}$ shorter than women in Zambia. Lower heights could lead to smaller pelvic girdles, which may lead to a higher incidence of fetal growth restriction and obstructed labour [26]. Thus, it would be in the best interest of the fetus to deliver earlier to avoid complications, leading to the argument that shorter gestational age may be an evolutionary adaptation in mothers of some ethnicities [27]. A study suggests anatomic constraints play a more significant role than genetics on premature birth [28].

Our findings on the risk factors of preterm birth corroborate well with previous studies [29,30]. In our study, multiple gestations were the strong risk factor for preterm births. Other risk factors associated with preterm birth included antepartum haemorrhage, pre-eclampsia/ eclampsia, and fever, while clean fuel availability was found to be protective. Three-quarters of children born live before 28 weeks of completed gestation died before completing the first 28 days of life. The risk of mortality decreased subsequently with increasing gestational age, eventually rising slightly in the post-term group ( $\geq 42$ weeks). This is consistent with previous literature [2].

Our finding highlighted that preterm birth is the key to reduce the neonatal mortality rate further. As many as one in two neonatal deaths occurred in neonates who were born too soon. Furthermore, we observed a high level of mortality in premature infants (14\%), many of which could have survived in high-income countries (HIC). In HIC, more than $90 \%$ of preterm babies born $<28$ weeks survive, while in LMICs, they die in the first few days [31]. These findings draw urgent attention to provide adequate and timely intervention, such as antenatal steroid injections for mothers in premature labour, Kangaroo Mother Care (KMC) for preterm /ow birth weight babies immediately after birth with respiratory support. A recent study showed that antenatal steroid injection improved neonatal deaths by 16\% [32]. Another study showed a 25\% reduction in neonatal deaths if KMC was initiated as soon as possible after birth [33]. These simple and inexpensive interventions could save newborn lives.

Assessing accurate gestational age is the first step to identify preterm birth and providing effective measures. In our study, regional differences in the burden of preterm birth disappeared when we used LMP to calculate gestational age. This points towards measurement error of using LMP to estimate gestational age and can be the reason for inconsistencies reported previously in the literature [34]. In our study, we observed a wide limit of agreement between the two measures (-35.2 to 30.1) and that LMP tends to overestimate early gestation and underestimate late gestation, misclassifying term and preterm births in setting where ultrasound access is limited. This implies that preterm newborns may not receive the appropriate level of care if not identified in a timely manner, especially in the early days of life. Therefore, the priority to improve classification and identification of preterm neonates should be to ensure adequate investment in obstetric ultrasound scans in early pregnancy. Concurrently, in a setting where access to ultrasound is limited, we have developed new machine learning models combining newborn characteristics including anthropometrics or LMP, that may predict gestational age within $\pm 15.7-18.4$ days of early ultrasound dating. These methods need further testing and could potentially serve as an alternative to early ultrasound dating in low resource settings [35].

Our study highlighted the importance of ensuring women with multiple gestations to be identified early by ultrasound and checked for any potential risk for PROM and other obstetric complications. In addition, com- 
prehensive reproductive history, such as women's previous history of preterm birth and stillbirths, needs to be examined for recurrent preterm birth. Prevention and diagnosis of maternal morbidity such as pre-eclampsia will help reduce preterm labour. All pregnant adolescents, especially with multiple gestations, should receive appropriate and timely antenatal care, including contraception use and health education activities. Poverty was a risk factor for preterm birth in our study. Lower socioeconomic status, in general, continues to influence developmental delay at two years of age for neonates born early and poor [36]. Programs to focus on early childhood development by improved parenting skills and nurturing the home environment can mitigate the effect of poverty [36].

\section{Strengths and limitations}

There are a few strengths of this study. This study was conducted in five sites in South-Asia and sub-Saharan Africa, which bear the highest burden of adverse birth outcomes. There, high-quality vital information on birth and deaths is sparse or non-existent. Second, this was a large population-based study in which pregnancies are identified by household surveillance of women of reproductive age. The sites also had a birth notification system to ensure early detection and identification of birth outcomes, resulting in low loss to follow up. Third, we used rigorous training to standardize sonographers to performs first-trimester ultrasound dating in all sites. Lastly, we were also able to compare two different methods for calculating gestational age, ie, LMP and ultrasound.

We had a few limitations. First, anthropometric measurements, including the height and weight of the mothers, were not available for all women, which would have been helpful to examine the association of maternal BMI and preterm births. In Pakistan site, in particular, height measurements were available for only one-third of the women. Second, although this was a large population-based study with higher precision on the results, these results may not fully represent the entire country. All study sites are predominantly rural areas except Pakistan (Karachi) site that is peri-urban. Third, we enrolled women presenting at the antenatal care clinic before 20 weeks of gestation. This could lead to a selection bias if women seek antenatal care later in pregnancy have a higher or lower risk of preterm birth, however by study design, we used early ultrasound dating to have accurate gestational age dating.

\section{CONCLUSIONS}

A population-based cohort study showed that the incidence of preterm birth is much higher in South-Asian sites than in sub-Saharan African sites. High rates of neonatal mortality among preterm births, particularly in very and moderate preterm babies, calls for urgent attention to developing policies and intervention packages to improve care around birth and early identification of high-risk pregnancies.

\section{AMANHI GA Study Group collaborators:}

Fahad Aftab (Centre for Public Health Kinetics, New Delhi, India), Parvez Ahmed (Institute of Epidemiology Disease Control and Research, Dhaka, Bangladesh), Salahuddin Ahmed (Projahnmo Research Foundation, Dhaka, Bangladesh), Corneille Bashagaluke Akonkwa (McGill University Health Centre, Montreal, Québec, Canada), Said Mohammed Ali (Public Health Laboratory Ivo de Carneri, Pemba, Tanzania), Rajiv Bahl (Department of Maternal, Newborn, Child and Adolescent Health and Ageing, WHO, Geneve, Switzerland), Bowen Banda (Research Unit of Environmental Sciences and Management, North-West University, Potchefstroom, South Africa), Abdullah H Baqui (Department of International Health, Johns Hopkins University Bloomberg School of Public Health, Baltimore, Maryland, USA), Nazma Begum (Projahnmo Research Foundation, Dhaka, Bangladesh), Godfrey Biemba (National Health Research Authority, Lusaka, Zambia), Sayan Das (Center for Public Health Kinetics, New Delhi, India), Saikat Deb (Public Health Laboratory Ivo de Carneri, Pemba, Tanzania), Usha Dhingra (Center for Public Health Kinetics, New Delhi, India), Arup Dutta (Center for Public Health Kinetics, New Delhi, India), Karen Edmond (Maternal and Child Health, King's College London, London, UK), Davidson H Hamer (Department of Global Health, Boston University School of Public Health, Boston, Massachusetts, USA; Section of Infectious Diseases, Department of Medicine, Boston University School of Medicine, Boston, Massachusetts, USA), Julie Herlihy (Department of Pediatrics, Boston University School of Medicine, Boston, Massachusetts, USA ), Lisa Hurt (Division of Population Medicine, Cardiff University School of Medicine, Cardiff, UK), Fyezah Jehan (Department of Paediatrics and Child Health, The Aga Khan University, Karachi, Pakistan), Mohamed Hamad Juma (Public Health Laboratory Ivo de Carneri, Pemba, Tanzania), Monica Lulu Kapasa (Pediatrics, University of Zambia, Lusaka, Zambia), Muhammad Karim (The Aga Khan University, Karachi, Sindh, Pakistan), Farzana Kausar (The Aga Khan University, Karachi, Sindh, Pakistan), Farah Khalid (The Aga Khan University, Karachi, Sindh, Pakistan), Betty R Kirkwood (Epidemiology and Population Health, London School of Hygiene \& Tropical Medicine, London, UK), Anne CC Lee (Global Advancement of Infant and Maternal Health Laboratory, Brigham and Women's Hospital, Boston, Massachusetts, USA; Harvard Medical School, Boston, Massachusetts, USA), 
Alexander Manu (University of Ghana School of Public Health, Accra, Greater Accra, Ghana), Usma Mehmood (The Aga Khan University, Karachi, Sindh, Pakistan), Dipak Mitra (Department of Public Health, North South University, Dhaka, Bangladesh), Fern Mweene (Kazungula District Hospital, Kazungula, Zambia), Naila Nadeem (The Aga Khan University, Karachi, Sindh, Pakistan), Muhammad Imran Nisar (The Aga Khan University, Karachi, Sindh, Pakistan), Rina Paul (Center for Non-communicable Diseases and Nutrition, BRAC University James P Grant School of Public Health, Dhaka, Bangladesh), Mahmoodur Rahman (Maternal and Child Health Division, International Centre for Diarrhoeal Disease Research Bangladesh, Dhaka, Bangladesh), Sayedur Rahman (Projahnmo Research Foundation, Dhaka, Bangladesh), Muhammad Sajid (The Aga Khan University, Karachi, Sindh, Pakistan), Sunil Sazawal (Center for Public Health Kinetics, New Delhi, India), Katherine E Semrau (Ariadne Laboratories, Harvard T.H Chan School of Public Health, Division of Global Health Equity, Brigham and Women's Hospital, Boston, Massachusetts, USA; Department of Medicine, Harvard Medical School, Boston, Massachusetts, USA), Shahira Shahid (The Aga Khan University, Karachi, Sindh, Pakistan), Caitlin Shannon (CARE USA, New York, New York, USA), Marina Straszak-Suri (Obstetrics and Gynecology, University of Ottawa, Ottawa, Ontario, Canada), Atifa Suleiman (Public Health Laboratory Ivo de Carneri, Pemba, Tanzania), Mohammad J Uddin (Save the Children Bangladesh, Dhaka, Bangladesh), Jayson Wilbur (Metrum Research Group, Tariffville, Connecticut, USA), Blair Wylie (Harvard Medical School, Boston, Massachusetts, USA; Division of Maternal-Fetal Medicine, Beth Israel Deaconess Medical Center, Boston, Massachusetts, USA), Sachiyo Yoshida (Department of Maternal, Newborn, Child and Adolescent Health and Ageing, WHO, Geneve, Switzerland).

\footnotetext{
Acknowledgements: We would like to thank all of the pregnant women and infants who participated in the study. We
acknowledge all research field teams and communities at each site.
Funding: This study is part of the AMANHI initiative funded by the Bill \& Melinda Gates Foundation via OPP1054163
and coordinated through the World Health Organization MCA department. Funders had no roles in the collection, anal-
ysis, or interpretation of the results.
Authorship contributions: The study was conceptualized and designed by principal investigators of the five sites: AB
(Bangladesh), DH \& KES (Zambia), FJ (Pakistan), BK (Ghana), SS (Tanzania)] and ACL, LH, CS, AM, IN, SA, UD, GB, JH,
and RB. All authors from five sites (Bangladesh, Ghana, Pakistan, Tanzania, Zambia) conducted the acquisition of data. BW
led the oversight and quality control in ultrasonography in the study and SY, TD, NM and RB conducted the analysis. IN
wrote the first draft of the manuscript. All authors discussed the results in the workshop and contributed to the revision of
the final manuscript. All listed authors meet authorship criteria and that no others meeting the criteria have been omitted.
Competing interests: The WHO and study sites received funding from the Bill and Melinda Gates Foundation (BMGF)
to conduct this study. BB, CBA, DHH, JH, LH, FM, KES, MSS and JW report research grants from the BMGF during the
conduct of the study. ACL reported research grants from the NICHD and BMGF. The authors completed the ICMJE Decla-
ration of Interest form (available upon request from the corresponding authors), and declare no further conflicts of interest.
Additional material
Online Supplementary Document
}

1 Chawanpaiboon S, Vogel JP, Moller AB, Lumbiganon P, Petzold M, Hogan D, et al. Global, regional, and national estimates of levels of preterm birth in 2014: a systematic review and modelling analysis. Lancet Glob Health. 2019;7:e37-46. Medline:30389451 doi:10.1016/S2214-109X(18)30451-0

2 Blencowe H, Cousens S, Chou D, Oestergaard M, Say L, Moller AB, et al. Born too soon: the global epidemiology of 15 million preterm births. Reprod Health. 2013;10 Suppl 1:S2. Medline:24625129 doi:10.1186/1742-4755-10-S1-S2

$3 \mathrm{WHO}$. recommended definitions, terminology and format for statistical tables related to the perinatal period and use of a new certificate for cause of perinatal deaths. Modifications recommended by FIGO as amended October 14, 1976. Acta Obstet Gynecol Scand. 1977;56:247-53. Medline:560099

4 ACOG Committee Opinion No 579. Definition of term pregnancy. Obstet Gynecol. 2013;122:1139-40. Medline:24150030 doi:10.1097/01.AOG.0000437385.88715.4a

5 Mustafa G, David RJ. Comparative accuracy of clinical estimate versus menstrual gestational age in computerized birth certificates. Public health reports (Washington, DC: 1974). 2001;116:15-21.

6 Alexander GR, Tompkins ME, Petersen DJ, Hulsey TC, Mor J. Discordance between LMP-based and clinically estimated gestational age: implications for research, programs, and policy. Public Health Rep. 1995;110:395-402. Medline:7638326

7 Salomon LJ, Alfirevic Z, Bilardo CM, Chalouhi GE, Ghi T, Kagan KO, et al. ISUOG practice guidelines: performance of first-trimester fetal ultrasound scan. Ultrasound Obstet Gynecol. 2013;41:102-13. Medline:23280739 doi:10.1002/uog.12342

8 Hoffman CS, Messer LC, Mendola P, Savitz DA, Herring AH, Hartmann KE. Comparison of gestational age at birth based on last menstrual period and ultrasound during the first trimester. Paediatr Perinat Epidemiol. 2008;22:587-96. Medline:19000297 doi:10.1111/j.1365-3016.2008.00965.x

9 Lynch CD, Zhang J. The research implications of the selection of a gestational age estimation method. Paediatr Perinat Epidemiol. 2007;21 Suppl 2:86-96. Medline:17803622 doi:10.1111/j.1365-3016.2007.00865.x 
10 Ballard JL, Khoury JC, Wedig K, Wang L, Eilers-Walsman BL, Lipp R. New Ballard Score, expanded to include extremely premature infants. J Pediatr. 1991;119:417-23. Medline:1880657 doi:10.1016/S0022-3476(05)82056-6

11 Engstrom JL. Measurement of Fundal Height. J Obstet Gynecol Neonatal Nurs. 1988;17:172-8. Medline:3292729 doi:10.1111/j.1552-6909.1988.tb00422.x

12 Dubowitz LM, Dubowitz V, Goldberg C. Clinical assessment of gestational age in the newborn infant. J Pediatr. 1970;77:1-10. Medline:5430794 doi:10.1016/S0022-3476(70)80038-5

13 Kramer MS, McLean FH, Boyd ME, Usher RH. The validity of gestational age estimation by menstrual dating in term, preterm, and postterm gestations. JAMA. 1988;260:3306-8. Medline:3054193 doi:10.1001/jama.1988.03410220090034

14 Rosenberg RE, Ahmed ASMNU, Ahmed S, Saha SK, Chowdhury MAKA, Black RE, et al. Determining gestational age in a low-resource setting: validity of last menstrual period. J Health Popul Nutr. 2009;27:332-8. Medline:19507748

15 Goldenberg RL, Culhane JF, Iams JD, Romero R. Epidemiology and causes of preterm birth. Lancet. 2008;371:75-84. Medline:18177778 doi:10.1016/S0140-6736(08)60074-4

16 Dekker GA, Lee SY, North RA, McCowan LM, Simpson NA, Roberts CT. Risk factors for preterm birth in an international prospective cohort of nulliparous women. PLoS One. 2012; 7:e39154. Medline:22815699 doi:10.1371/journal.pone.0039154

17 Chang HH, Larson J, Blencowe H, Spong CY, Howson CP, Cairns-Smith S, et al. Preventing preterm births: analysis of trends and potential reductions with interventions in 39 countries with very high human development index. Lancet. 2013;381:22334. Medline:23158883 doi:10.1016/S0140-6736(12)61856-X

18 Tunçalp Ö, Pena-Rosas JP, Lawrie T, Bucagu M, Oladapo OT, Portela A, et al. WHO recommendations on antenatal care for a positive pregnancy experience-going beyond survival. BJOG. 2017;124:860-2. Medline:28190290 doi:10.1111/1471-0528.14599

19 Baqui A, Ahmed P, Dasgupta SK, Begum N, Rahman M, Islam N, et al. Development and validation of a simplified algorithm for neonatal gestational age assessment - protocol for the Alliance for Maternal Newborn Health Improvement (AMANHI) prospective cohort study. J Glob Health. 2017;7:021201. Medline:29163937 doi:10.7189/jogh.07.021201

20 Papageorghiou AT, Kennedy SH, Salomon LJ, Altman DG, Ohuma EO, Stones W, et al. The INTERGROWTH-21(st) fetal growth standards: toward the global integration of pregnancy and pediatric care. Am J Obstet Gynecol. 2018;218 2s:S630-S640. Medline:29422205 doi:10.1016/j.ajog.2018.01.011

21 Victora CG, Huttly SR, Fuchs SC, Olinto MTA. The Role of Conceptual Frameworks in Epidemiological Analysis: A Hierarchical Approach. Int J Epidemiol. 1997;26:224-27. Medline:9126524 doi:10.1093/ije/26.1.224

22 Imdad A, Bhutta ZA. Maternal nutrition and birth outcomes: effect of balanced protein-energy supplementation. Paediatr Perinat Epidemiol. 2012;26 Suppl 1:178-90. Medline:22742610 doi:10.1111/j.1365-3016.2012.01308.x

23 Haider BA, Bhutta ZA. Multiple-micronutrient supplementation for women during pregnancy. Cochrane Database Syst Rev. 2017:4:CD004905. Medline:28407219

24 Kozuki N, Katz J, Lee AC, Vogel JP, Silveira MF, Sania A, et al. Short Maternal Stature Increases Risk of Small-for-Gestational-Age and Preterm Births in Low- and Middle-Income Countries: Individual Participant Data Meta-Analysis and Population Attributable Fraction. J Nutr. 2015;145:2542-50. Medline:26423738 doi:10.3945/jn.115.216374

25 Khanam R, Lee AC, Mitra DK, Ram M, Das Gupta S, Quaiyum A, et al. Maternal short stature and under-weight status are independent risk factors for preterm birth and small for gestational age in rural Bangladesh. Eur J Clin Nutr. 2019;73:733-42. Medline:29934512 doi:10.1038/s41430-018-0237-4

26 Christian P, Lee SE, Donahue Angel M, Adair LS, Arifeen SE, Ashorn P, et al. risk of childhood undernutrition related to small-for-gestational age and preterm birth in low- and middle-income countries. Int J Epidemiol. 2013;42:1340-55. Medline:23920141 doi:10.1093/ije/dyt109

27 Patel RR, Steer P, Doyle P, Little MP, Elliott P. Does gestation vary by ethnic group? A London-based study of over 122,000 pregnancies with spontaneous onset of labour. Int J Epidemiol. 2004;33:107-13. Medline:15075154 doi:10.1093/ije/dyg238

28 Zhang G, Bacelis J, Lengyel C, Teramo K, Hallman M, Helgeland $\varnothing$, et al. Assessing the Causal Relationship of Maternal Height on Birth Size and Gestational Age at Birth: A Mendelian Randomization Analysis. PLoS Med. 2015;12:e1001865. Medline:26284790 doi:10.1371/journal.pmed.1001865

29 Malley CS, Kuylenstierna JC, Vallack HW, Henze DK, Blencowe H, Ashmore MR. Preterm birth associated with maternal fine particulate matter exposure: A global, regional and national assessment. Environ Int. 2017;101:173-82. Medline:28196630 doi:10.1016/j.envint.2017.01.023

30 Stewart A, Graham E. Preterm birth: An overview of risk factors and obstetrical management. Dev Disabil Res Rev. 2010;16:2858. Medline:25708070 doi:10.1002/ddrr.124

31 March of Dimes. PMNCH, Save the Children, WHO. Born Too Soon: The Global Action Report on Preterm Birth. Eds CP Howson, MV Kinney, JE Lawn. Geneva: World Health Organization; 2012.

32 WHO Immediate KMC Study Group. Immediate "Kangaroo Mother Care" and Survival of Infants with Low Birth Weight. N Engl J Med. 2021;384:2028-38. Medline:34038632 doi:10.1056/NEJMoa2026486

33 WHO ACTION Trials Collaborators. Antenatal Dexamethasone for Early Preterm Birth in Low-Resource Countries. N Engl J Med. 2020;383:2514-25. Medline:33095526 doi:10.1056/NEJMoa2022398

34 Hall ES, Folger AT, Kelly EA, Kamath-Rayne BD. Evaluation of gestational age estimate method on the calculation of preterm birth rates. Matern Child Health J. 2014;18:755-62. Medline:23775254 doi:10.1007/s10995-013-1302-1

35 Alliance for Maternal and Newborn Health Improvement (AMANHI) Gestational Age Study Group; Alliance for Maternal and Newborn Health Improvement (AMANHI) GA Study Group. Simplified models to assess newborn gestational age in low-middle income countries: findings from a multi-country, prospective cohort study. BMJ Glob Health. 2021;6:e005688. Medline:34518201 doi:10.1136/bmjgh-2021-005688

36 Brumberg HL, Shah SI. Born early and born poor: An eco-bio-developmental model for poverty and preterm birth. J Neonatal Perinatal Med. 2015;8:179. Medline:26485551 doi:10.3233/NPM-15814098 